\title{
Phase-Rectified Signal Averaging for Automatic Detection of QRS Fragmentation
}

\author{
Griet Goovaerts $^{1,2}$, Bert Vandenberk ${ }^{3}$, Carolina Varon ${ }^{1,2}$, Rik Willems ${ }^{3}$, Sabine Van Huffel ${ }^{1,2}$ \\ ${ }^{1}$ KU Leuven, Department of Electrical Engineering-ESAT, STADIUS Centre for Dynamical \\ Systems, Signal Processing and Data Analytics, Leuven, Belgium. \\ 2 iMinds, Medical Information Technologies, Leuven, Belgium \\ ${ }^{3}$ KU Leuven, Division of Experimental Cardiology, Department of Cardiovascular Diseases, Leuven, \\ Belgium
}

\begin{abstract}
QRS fragmentation is visible in the ECG signal as the presence of one or more deflections, notches or slurs in the QRS complex. The presence of QRS fragmentation is strongly related with the myocardial fibrosis or scarrings and has been associated with adverse outcome in patients. Since detection of fragmented QRS complexes is mainly done on a visual basis, its practical use is limited. We propose an automatic method to detect the QRS fragmentation based on Phase-Rectified Signal Averaging (PRSA). The method calculates the PRSA curve, approximates it with a linear fit and derives four parameters related to the slope and linear fit. Analysis of a dataset from the University Hospitals of Leuven with 268 patients shows that all four parameters are significantly different in fragmented channels compared to normal channels $(p<0.001)$. Furthermore, Cohen's $d$ suggests that three out of four parameters have at least a large effect size $(d>0.8)$.
\end{abstract}

\section{Introduction}

The timing and morphology of the QRS complex is a widely-studied subject since the 1960s [1]. Changes in the morphology and specific morphology patterns can be important tools in the diagnosis of myocardial fibrosis or scars [2]. Fragmented QRS complexes (fQRS) in a QRS complex with normal duration $(<120 \mathrm{~ms})$ are defined as a QRS complex that contains one or more deflections, notches or slurs. Examples of QRS fragmentation can be seen in Figure 1. In general, all shown types of QRS variations are considered fQRS.

The presence of QRS fragmentation is a known indicator for myocardial scarring [2], and has been shown to predict cardiac events in patients with coronary artery disease and (non-)ischemic cardiomyopathy [3,4]. Currently, detection of QRS fragmentation in clinical practice is done on a visual basis by inspecting the ECG signal lead-by-lead. This is a time-consuming task that is sensitive to subjective interpretation of the ECG signals. Automated detection methods would remove these limitations, benefiting the clinical usefulness of this parameter.

We propose a fully automatic method that applies PhaseRectified Signal Averaging (PRSA) to detect fragmented QRS complexes. PRSA is a technique that detects quasiperiodicities in non-stationary signals, and is often used to characterize the deceleration and acceleration capacity of the heart starting from the tachogram [5]. We show that PRSA can also be applied directly to the ECG signal to detect fQRS.

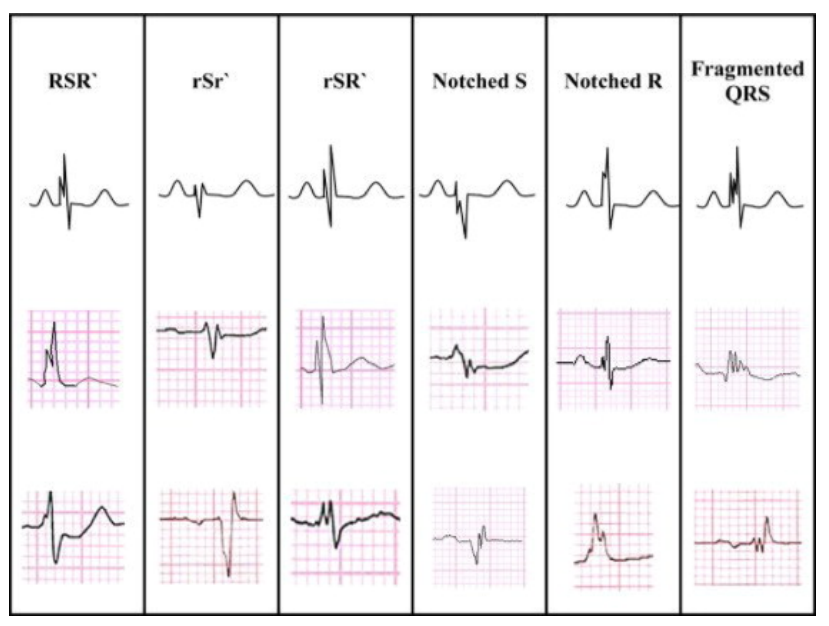

Figure 1: Examples of different types of fragmented QRS complexes (reprinted from [4]). 


\section{Data and methods}

\subsection{Data}

The dataset for this study has been measured in the University Hospital Leuven. It consists of clinical 12-lead ECGs of 268 patients who received an Implantable Cardiac Defibrillator (ICD). Selection criteria were a normal sinus rhythm and normal QRS duration $(<120 \mathrm{~ms})$. All signals are sampled at $200 \mathrm{~Hz}$ and have a duration of $10 \mathrm{~s}$. The dataset is fully annotated by an experienced cardiologist, meaning that each lead of each signal was scored on the presence of QRS fragmentation.

\subsection{Preprocessing}

Since the clinical ECGs are relatively noisefree, little preprocessing is necessary. The signals are first upsampled to $2000 \mathrm{~Hz}$ by linearly interpolating the samples in order to increase the number of samples in a QRS complex. This will facilitate parameter selection and linear fitting later. In a second step they are normalized channel-by-channel by calculating the $\mathrm{z}$-score to remove amplitude differences among the channels. The R peaks are detected using PanTompkins. Finally, QRS segmentation is done by selecting a window of $100 \mathrm{~ms}$ around each $\mathrm{R}$ peak.

\subsection{Phase-Rectified Signal Averaging}

Phase-Rectified Signal Averaging typically consists of three basic steps. The first step is the selection of anchor points $x_{i}$. Anchor points are points on the ECG signal that meet a certain criterion. Typical criteria are based on (average) increases and decreases in the signal:

$$
\begin{aligned}
& \frac{1}{T} \sum_{j=0}^{T-1} x_{i+j}>\frac{1}{T} \sum_{j=1}^{T} x_{i-j} \\
& \frac{1}{T} \sum_{j=0}^{T-1} x_{i+j}<\frac{1}{T} \sum_{j=1}^{T} x_{i-j}
\end{aligned}
$$

In these Equations, $T$ is a parameter that can be used to compare averages of a period of $T$ samples. $T$ effectively functions as a low-pass filter that limits the upper frequency of the periodicities that will be detected. Here, the selected anchor points are all points that are located on an increasing part of the QRS complex. This corresponds to points that meet Equation 1 with $T=1$;

In the second step, windows of length $2 L$ are selected surrounding each anchor point. We disregard sample points that are close ( $<L$ samples) to the beginning or the end of the QRS complex, since for these samples no such windows can be selected. $L$ has to be chosen carefully, since

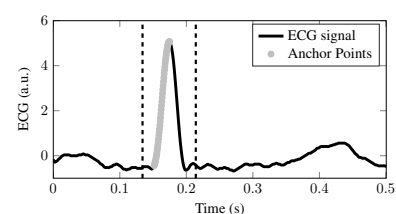

(a) Anchor Point (AP) selection.

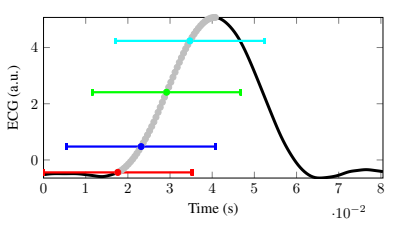

(b) AP window selection.

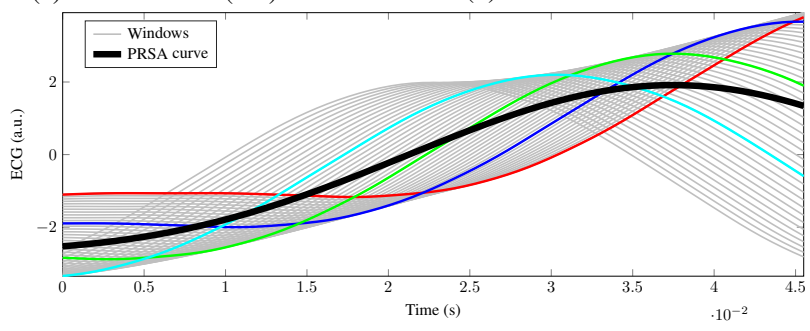

(c) Alignment of windows and calculation of PRSA curve.

Figure 2: Illustration of the three different steps of PRSA applied to one heartbeat of the ECG signal.

it has to be larger than the slowest periodicity that is expected to occur. Here, $L$ is equal to 45 . Finally, the different windows around all anchor points are then aligned and averaged, obtaining the PRSA curve $\bar{x}_{k}$. Figure $3 \mathrm{~b}$ illustrates the different steps of the algorithm.

Normal QRS complexes have a very simple morphology consisting of one increasing and one decreasing part. When PRSA is applied to these complexes, approximately half of the samples will be selected as anchor points and these points will be located in one part of the QRS complex (see Figure 2a). The corresponding windows will thus largely overlap each other and mainly cover the increasing part of the QRS complex. This is shown on Figure $2 b$, where 4 anchor points and their surrounding windows are indicated in color. The resulting PRSA curve of these anchor points (shown in Figure 2c) will be the average of these windows and can be approximated with a straight line. The APs of fragmented QRS complexes on the other hand will be distributed over the complete length of the QRS. Since the surrounding windows will not only cover the increasing part of the QRS complex but also parts of the decreasing part, the resulting PRSA curve will be less straight. Examples of (fragmented) QRS complexes and their PRSA curves are shown in Figure 3.

\subsection{Quantification of PRSA curve}

As explained in the previous paragraph, we can approximate the PRSA curve of a QRS complex with a linear fit:

$$
\bar{x}_{k} \approx a_{k} t+b_{k}
$$

To quantify the PRSA curve we define 4 different parameters, based on basic characteristics of the PRSA curve and its linear fit: 


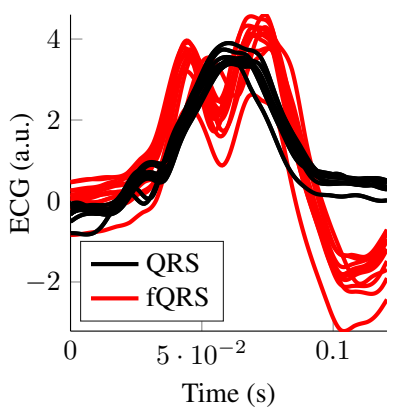

(a) QRS complexes

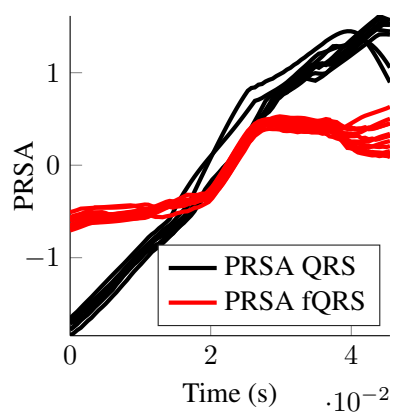

(b) PRSA curves
Figure 3: (a) Examples of QRS complexes in a normal channel (black) and a fragmented channel (red) and (b) their corresponding PRSA curves.

1. Mean derivative of PRSA curve: $\frac{1}{2 L} \sum_{t=0}^{2 L} \frac{d \bar{x}_{k}}{d t}$

2. Slope of linear fit $\left(a_{k}\right)$

3. Intercept of linear fit with y-axis $\left(b_{k}\right)$

4. Norm of residuals

Since the PRSA curve is calculated for each heartbeat and a decision about fragmentation is made channel-bychannel, the results for each channel are computed by taking the minimum value for all curves in one channel for parameters 1-3 and the maximal value for parameter 4 (norm of residuals).

\subsection{Statistical tests}

The differences between normal channels and channels containing fQRS are tested with a two-sample t-test. The significance level is set to $p=0.001$. In addition to a pvalue, an estimate of the effect size is also calculated using Cohen's d [6]:

$$
d=\frac{\mu_{1}-\mu_{2}}{\sqrt{\left(\sigma_{1}^{2}+\sigma_{2}^{2}\right) / 2}}
$$

$\mu_{1}, \mu_{2}$ and $\sigma_{1}, \sigma_{2}$ are respectively the mean and standard deviation of both groups. Effect sizes can be classified using $d$-values as small $(\mathrm{d}>0.2)$, medium $(\mathrm{d}>0.5)$, large $(\mathrm{d}>0.8)$ and very large $(\mathrm{d}>1.3)$ [7].

\section{Results and discussion}

Figure 3a shows the QRS complexes of two channels, one with (red) and one without (black) QRS fragmentation. Visually, the fragmented QRS complexes are easily recognizable by the notch near the location of the $\mathrm{R}$ peak. The resulting PRSA curves can be seen on Figure $3 b$. The difference between both types is evident: the black PRSA curves corresponding to normal QRS complexes resemble a (straight) line while the fragmented QRS produce a flatter trajectory. The complete dataset contains 2519 normal

\begin{tabular}{l|ccc} 
Parameter & fQRS & QRS & d \\
\hline Mean derivative & $0.003 \pm 0.004$ & $0.006 \pm 0.003$ & 0.88 \\
Slope & $0.005 \pm 0.004$ & $0.008 \pm 0.003$ & 0.78 \\
Intercept & $0.25 \pm 0.16$ & $0.38 \pm 0.16$ & 0.81 \\
Norm residuals & $1.1 \pm 0.55$ & $0.38 \pm 0.28$ & 1.62 \\
\hline
\end{tabular}

Table 1: Mean values and standard deviations of all parameters in fragmented $(f Q R S)$ and normal channels $(Q R S)$. d is the corresponding value for Cohen's d. All p-values obtained by a two-sample t-test were significant $(p<0.001)$ and are therefore not specifically mentioned.

channels and 591 channels with fragmentation. The results for the different parameters from Section 2.4 are summarized in Table 1. After statistical analysis, all p-values were significant $(p<0.001)$, therefore they are not explicitly mentioned. Analysis of Cohen's d shows that all parameters have at least moderate effect size. The mean derivative and the intercept have a large effect size, and the norm of the residuals has a d-value of 1.62, indicating a very large effect size or a very large practical significance. Figure 4 , where the values from Table 1 are shown as boxplots, confirms these findings: the mean values of all parameters differ between both groups, but the values for the norm residuals show the most obvious contrast. This confirms the large d-value of this parameter. Note that the standard deviations for especially the first two parameters are rather high for both groups. Further research is necessary to determine whether this is caused by particularly noisy ECG signals or normal variation in QRS morphology. Another possibility is optimizing the method to combine the results from different heartbeats to a result for the complete channel. Currently this is done by simply taking the minimum or maximum value of a parameter in that channel. If a channel contains an outlier (caused by for example noise or incorrect $\mathrm{R}$ peak detection) this outlier will be selected and may influence the results. A more advanced selection criterion that fuses multiple values will reduce this effect. Since $\mathrm{fQRS}$ is often examined per cardiac region in clinical practice, this method can then also be used as a mechanism to combine the results for a single channel to one value for each region.

The obtained results clearly show that characterization of the PRSA curve by means of a linear fit is an effective way to detect QRS fragmentation. Since the parameters used in the analysis are continuous parameters they can also be used as a form of fQRS quantification. To confirm this however, a more extensive study using more detailed annotations is necessary. The next step is to extend the dataset with a healthy control population, to verify whether parameter values of normal QRS complexes in the ICD group are comparable to truly healthy signals. This way a threshold can be determined to obtain a true fQRS detec- 


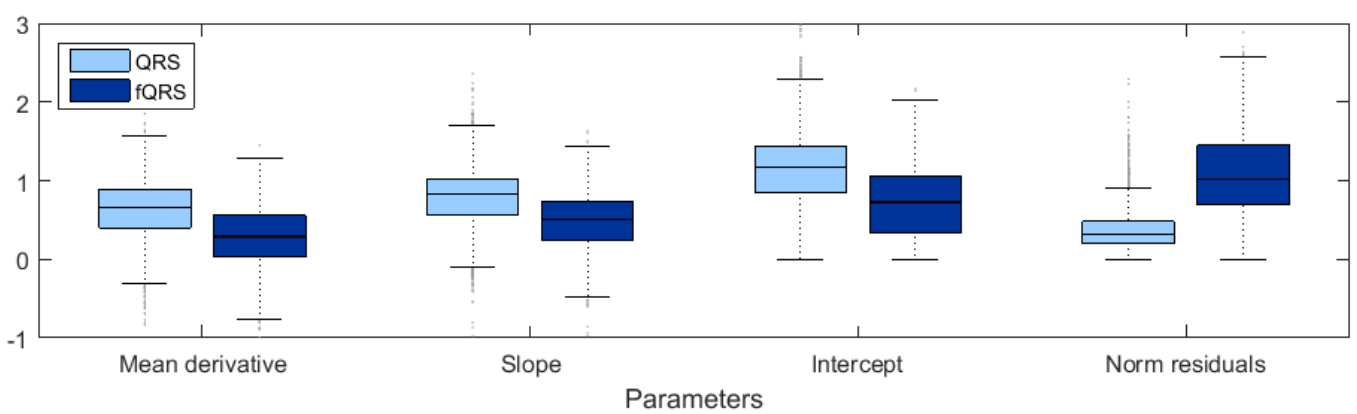

Figure 4: Boxplots for all 4 parameters, indicating the median, 25th and 75th quantile for both groups (normal QRS in light blue and fragmented QRS in dark blue).

tor that can be implemented in the hospital to establish the correlation of these results with cardiac events and clinical outcome.

\section{Conclusion}

In this paper we present an automatic method for detection of fragmented QRS complexes. Phase-Rectified Signal Averaging is applied to the QRS complex, and 4 parameters are derived from the resulting curve. Analysis of an annotated database shows significant differences for all parameters. The obtained results prove that the proposed method is a powerful way to detect fQRS and further research will decide whether the parameters can also be used to quantify fQRS as opposed to merely detecting its presence. The development of an automated method anyhow eliminates the need for visual inspection of the ECG signal, improving its practical use in day-to-day practice.

\section{Acknowledgements}

Research supported by Research Council KU Leuven (BOF): CoE PFV/10/002 (OPTEC); FWO: G.0427.10N (Integrated EEG-fMRI), G.0108.11 (Compressed Sensing), G.0869.12N (Tumor imaging), G.0A5513N (Deep brain stimulation); IWT: TBM 080658-MRI (EEG-fMRI), TBM 110697-NeoGuard; iMinds: Dotatie-Strategisch basis onderzoek (SBO-2015), ICON: NXT_ Sleep; Belgian Federal Science Policy Office: IUAP P7/19/ (DYSCO, 'Dynamical systems, control and optimization', 20122017); Belgian Foreign Affairs-Development Cooperation: VLIR UOS programs (2013-2019); EU: FP7/20072013, EU MC ITN TRANSACT 2012, 316679, ERASMUS EQR: Community service engineer, 539642-LLP-12013, INTERREG IVB NWE programme RECAP 209G; ERC Advanced Grant, 339804 BIOTENSORS This paper reflects only the authors' views and the Union is not liable for any use that may be made of the contained information. Griet Goovaerts is supported by an IWT PhD grant.

\section{References}

[1] FLOWERS NC, HORAN LG, Thomas J, TOLLESON WJ. The anatomic basis for high-frequency components in the electrocardiogram. Circulation 1969;39(4):531-539.

[2] Das MK, Suradi H, Maskoun W, Michael MA, Shen C, Peng J, Dandamudi G, Mahenthiran J. Fragmented wide qrs on a 12-lead ecg a sign of myocardial scar and poor prognosis. Circulation Arrhythmia and Electrophysiology 2008; 1(4):258-268.

[3] Pietrasik G, Zareba W. Qrs fragmentation: diagnostic and prognostic significance. Cardiol J 2012;19(2):114-21.

[4] Das MK, Saha C, El Masry H, Peng J, Dandamudi G, Mahenthiran J, McHenry P, Zipes DP. Fragmented qrs on a 12-lead ecg: a predictor of mortality and cardiac events in patients with coronary artery disease. Heart Rhythm 2007; 4(11):1385-1392.

[5] Bauer A, Kantelhardt JW, Bunde A, Barthel P, Schneider R, Malik M, Schmidt G. Phase-rectified signal averaging detects quasi-periodicities in non-stationary data. Physica A Statistical Mechanics and its Applications 2006;364:423434.

[6] Cohen J. A power primer. Psychological bulletin 1992; 112(1): 155

[7] Sullivan GM, Feinn R. Using effect size-or why the $p$ value is not enough. Journal of graduate medical education 2012; 4(3):279-282.

Address for correspondence:

Griet Goovaerts

Kasteelpark Arenberg 10 - bus 2446

3001 Leuven, Belgium

griet.goovaerts@esat.kuleuven.be 